\title{
FINITE GROUPS OF SEITZ TYPE
}

\author{
MICHAEL ASCHBACHER
}

(Communicated by Pham Huu Tiep)

\begin{abstract}
We show that a useful condition of Seitz on finite groups of Lie type over fields of order $q>4$ is often satisfied when $q$ is 2 or 3 . We also observe that various consequences of the Seitz condition, established by Seitz and Cline, Parshall, and Scott when $q>4$, also hold when $q$ is 3 or 4 .
\end{abstract}

In this paper we explore a certain property shared by most finite groups of Lie type first exploited by Seitz in [Se1]. In order to state our results precisely we need to be a bit careful with notation and terminology.

So let $p$ be a prime, and define a Lie simple group of Lie type of characteristic $p$ to be a central factor group $\Omega$ of a group $\bar{G}_{\sigma}$, where $\bar{G}$ is a simply connected simple algebraic group over the algebraic closure $\overline{\mathbf{F}}_{p}$ of the field of order $p$ and $\sigma$ is a Steinberg endomorphism of $\bar{G}$ with finite fixed points. Thus $\Omega$ is a central factor group of a simply connected classical group $S L_{n}(q), S p_{n}(q), \operatorname{Spin}_{n}(q), S U_{n}(q)$, or of a simply connected exceptional group $G_{2}(q), F_{4}(q), \tilde{E}_{6}(q), \tilde{E}_{7}(q), E_{8}(q)$, ${ }^{3} D_{4}(q),{ }^{2} \tilde{E}_{6}(q),{ }^{2} B_{2}(q),{ }^{2} G_{2}(q)$, or ${ }^{2} F_{4}(q)$, for some power $q$ of $p$. In particular, $\Omega$ is quasisimple unless $\Omega$ is an image of $S L_{2}(2), S L_{2}(3), S U_{3}(2), S p_{4}(2), G_{2}(2)$, ${ }^{2} B_{2}(2),{ }^{2} G_{2}(3)$, or ${ }^{2} F_{4}(2)$. Moreover, we say that $\Omega$ is defined over $\mathbf{F}_{q}$.

A nearly simple group of Lie type of characteristic $p$ is a finite group $G$ possessing a normal subgroup $\Omega$, which is a Lie simple group of Lie type in characteristic $p$, and such that $C_{G}(\Omega)=Z(G)$ is a $p^{\prime}$-group.

In addition we adopt the following notation: Let $\Phi$ be a root system for $\Omega$, $\Phi^{+}$a positive system with simple system $\pi$, and $\left(\Omega, B_{\Phi}, N_{\Phi}, S\right)$ the Tits system determined by $\Phi$ and $\Phi^{+}$. Set $H_{\Phi}=B_{\Phi} \cap N_{\Phi}$, the Cartan subgroup of $\Omega$ determined by $\Phi$ and $\Phi^{+}, U=\operatorname{Rad}\left(B_{\Phi}\right)$, and for $\alpha \in \Phi$ let $U_{\alpha}$ be the root subgroup of $\alpha$.

Set $B=N_{G}(U)$; we call $B$ the Borel subgroup of $G$, and we call the overgroups of $B$ in $G$ the standard parabolic subgroups of $G$. The parabolic subgroups of $G$ are the conjugates of the standard parabolics. Recall that $U \in S y l_{p}(\Omega)$, so $G=\Omega B$ by a Frattini argument. Set $H=B \cap B^{w_{0}}$ and $N=N_{\Phi} H$, where $w_{0}$ is the long word in the Weyl group $W=N_{\Phi} / H_{\Phi} \cong N / H$.

Assume $q$ is a power of the prime $p, G$ is a nearly simple group of Lie type, $\Omega$ is quasisimple, defined over $\mathbf{F}_{q}$, and is not ${ }^{2} G_{2}(q)$, and $A u t_{G}(\Omega)$ is a group of inner-diagonal automorphisms of $\Omega$. We define such a group $G$ to be of Seitz type if

Received by the editors February 23, 2012 and, in revised form, March 7, 2012 and March 9, 2012.

2010 Mathematics Subject Classification. Primary 20D05, 20E42.

This work was partially supported by NSF grants DMS-0504852 and DMS-0969009. 
(Se1) for each $\alpha \in \Phi^{+}, H$ is irreducible on $U_{\alpha} / \Phi\left(U_{\alpha}\right)$ and $Z\left(U_{\alpha}\right)$, and

(Se2) for all distinct $\alpha, \beta$ in $\Phi^{+}$,

$$
C_{H}\left(U_{\alpha} / \Phi\left(U_{\alpha}\right)\right) \neq C_{H}\left(U_{\beta} / \Phi\left(U_{\beta}\right)\right) \neq C_{H}\left(Z\left(U_{\alpha}\right)\right) \neq C_{H}\left(Z\left(U_{\beta}\right)\right) .
$$

Recall that the odd dimensional unitary groups $\Omega$ can be thought of as having a root system of type BC with three root lengths; however, our convention will be that $\Omega$ has a root system of type $C_{l}$ with two root lengths, and with $U_{\alpha}$ nonabelian for $\alpha$ long.

Hidden in the proof of Lemma 3 of [Se1] is a sketch of a proof by Seitz that if $G$ is a nearly simple group of Lie type defined over $\mathbf{F}_{q}$ with $q>4$, then $G$ is of Seitz type, although the Seitz conditions (Se1) and (Se2) are implicit, rather than explicit, in Seitz' discussion. When $q=2, G$ is almost never of Seitz type. In this paper we fill in some of the details in Seitz' proof in the case $q>4$, and also treat the cases $q=3$ and $q=4$, showing when $q=4$ that only two groups are not of Seitz type and when $q=3$ that roughly half the groups are of Seitz type.

Theorem 1. Assume $G$ is a nearly simple group of Lie type defined over $\mathbf{F}_{q}$ such that $\Omega$ is quasisimple and not ${ }^{2} G_{2}(q)$, and $A u t_{G}(\Omega)$ is a group of inner-diagonal automorphisms of $\Omega$. Then one of the following holds:

(1) $G$ is of Seitz type.

(2) $q=2$.

(3) $q=4$ and $G / Z(G) \cong L_{3}(4)$ or $G_{2}(4)$.

(4) $q=3$ and $G / Z(G) \cong L_{4}(3), U_{4}(3)$, or $U_{4}(3)$ extended by $\mathbf{Z}_{2}$.

(5) $\Omega / Z(\Omega)$ is $P S p_{n}(3)$ with $n \geq 4, P \Omega_{m}^{\epsilon}(3)$ with $m \geq 7, F_{4}(3)$, or $G_{2}(3)$.

The Seitz condition has a number of useful consequences, some of which were established by Seitz in [Se1 and [Se2, and others by Cline, Parshall, and Scott in CPS, although under the hypothesis that $q>4$ rather than the Seitz conditions. We record some of these properties in section 1 , and sometimes include proofs, as formally our hypotheses are different.

Also when $q>4$ and $G$ is not $L_{2}(9)$, the fact that $G$ is of Seitz type, together with an argument of Ward in [W], can be used to show that the $p$-part of the Schur multiplier of a simple group $G$ of Lie type over $\mathbf{F}_{q}$ is trivial; see the final remark in section 1.

Elsewhere we explore the subgroup lattices of finite groups of Lie type, where various results can be proved using the fact that most groups of Lie type are of Seitz type. In particular, in A we use Theorem 1 to establish a certain property of maximal parabolics of finite groups of Lie type, and then in turn use that property in our program aimed at showing that certain finite lattices are not intervals in the subgroup lattices of finite groups.

\section{Properties of groups of Seitz type}

In this section we assume $p$ is a prime, $q$ is a power of $p, G$ is a nearly simple group of Lie type defined over $\mathbf{F}_{q}$ such that $\Omega$ is quasisimple and not ${ }^{2} G_{2}(q)$, and $A u t_{G}(\Omega)$ is a group of inner-diagonal automorphisms of $\Omega$. In addition we adopt the notation established in the introduction. For $X \leq G$, set $\Phi_{X}=\left\{\alpha \in \Phi: U_{\alpha} \cap X \neq 1\right\}$.

The purpose of this section is to record some facts about groups of Seitz type due to Seitz and Cline-Parshall-Scott in order to give the reader some feeling for why the Seitz hypothesis is of interest and for convenient reference in later papers. For example, the following result is essentially Lemma 3 in Se1: 
Lemma 1.1. Assume $G$ is of Seitz type and $X \leq U$ is $H$-invariant. Then

$$
X=\prod_{\alpha \in \Phi_{X}}\left(U_{\alpha} \cap X\right)
$$

and for each $\alpha \in \Phi_{X}, U_{\alpha} \cap X=U_{\alpha}$ or $Z\left(U_{\alpha}\right)$.

Lemma 1.2. Let $K$ be a parabolic subgroup of $G$ containing $H$. Then $H$ is contained in a Borel subgroup of $K$ so that $H$ is a Cartan subgroup of $K$.

Proof. This is 2.1 in $\mathrm{Se} 2$.

Lemma 1.3. If $C_{U}(H)=1$, then $N_{G}(H)=N$.

Proof. This is 2.3 in Se2.

Lemma 1.4. Assume $G$ is of Seitz type. Then $C_{U}(H)=1$.

Proof. Assume $C_{U}(H) \neq 1$. As $G$ is of Seitz type, it follows from Lemma 1.1 that $Z\left(U_{\alpha}\right) \leq C_{G}(H)$ for some $\alpha \in \Phi^{+}$. Let $l$ be the length of $\alpha$. As $N$ is transitive on roots of length $l$, it follows that $Z\left(U_{\beta}\right) \leq C_{G}(H)$ for each $\beta \in \Phi^{+}$of length $l$. Hence as $G$ is of Seitz type, $\alpha$ is the unique positive root of length $l$, so the Lie rank of $\Omega$ is 1 . Then it follows by inspection of the quasisimple groups of Lie rank 1 that $C_{U}(H)=1$.

The following result is due to Cline, Parshall, and Scott. Proofs appear in CPS and 2.4 of [Se2], but we also include a proof below, as our hypotheses are a bit different.

Theorem 1.5. Assume $G$ is of Seitz type and let $X$ be a nontrivial $H$-invariant p-subgroup of $\Omega$. Then

$$
X=\prod_{\alpha \in \Phi_{X}}\left(X \cap U_{\alpha}\right)
$$

and for each $\alpha \in \Phi_{X}, U_{\alpha} \cap X=U_{\alpha}$ or $Z\left(U_{\alpha}\right)$. In particular, $\left\{Z\left(U_{\alpha}\right): \alpha \in \Phi\right\}$ is the set of minimal nontrivial $H$-invariant p-subgroups of $\Omega$.

Proof. By the Borel-Tits Theorem, there is a parabolic subgroup $P$ of $G$ with $X \leq \operatorname{Rad}(P)$ and $N_{G}(X) \leq P$. Then $H \leq N_{G}(X) \leq P$, so by Lemma $1.2, H$ is contained in a Borel subgroup $B^{g}$ of $P$ for some $g \in G$. Now $H$ and $H^{g^{-1}}$ are Hall $p^{\prime}$-subgroups of $B$, so $H^{g^{-1}}=H^{u}$ for some $u \in U$. Then $n=u g \in N_{G}(H)=N$ by Lemmas 1.3 and 1.4 , so $B^{g}=B^{u g}=B^{n}$. Set $Y=X^{n^{-1}}$. By Lemma 1.1,

$$
Y=\prod_{\alpha \in \Phi_{Y}}\left(Y \cap U_{\alpha}\right)
$$

and $U_{\alpha} \cap Y=U_{\alpha}$ or $Z\left(U_{\alpha}\right)$ for each $\alpha \in \Phi_{Y}$. Then as $n$ acts on $\Phi$, the first statement in the lemma follows, while the second follows from the first.

Remark. Seitz uses Theorem 1.5 to give a description of the overgroups of $H$ in $G$ when $q>4$. His approach can be extended to all groups of Seitz type. Indeed in $[\mathrm{Se} 3$, Seitz considers maximal tori $T$ of $\Omega$ other than the Cartan subgroup $H$, defines $T$-root subgroups of $\Omega$, and shows in 5.3 of [Se3] that when $q>5$, distinct positive $T$-root subgroups are not $T$-isomorphic; that is, the $T$-root subgroups satisfy a Seitz condition. Then Seitz goes on to give a description of the overgroups of $T$ in $\Omega$. 
Part (2) of the next lemma is a result of Cline-Parshall-Scott in CPS. The proof of part (1) is essentially their proof of part (2).

Lemma 1.6. Assume $G$ is of Seitz type, and let $P$ be a parabolic subgroup of $G$, $R=\operatorname{Rad}(P)$, and $L$ a Levi factor of $P$. Then

(1) If $K \leq P$ with $P=R K$ and $Q=R \cap K \unlhd P$, then $K$ is conjugate under $R$ to $L Q$.

(2) All complements to $R$ in $P$ are Levi factors of $P$.

Proof. Observe that (2) is the special case of (1) where $Q=1$, so it remains to prove (1). Choose $K$ as in (1). Without loss of generality, $B \leq P$, and $L=\left\langle H, U_{\alpha}\right.$ : $\left.\alpha \in \Phi-\Phi_{R}\right\rangle$.

As $H$ is a $p^{\prime}$-group, $H$ is contained in $K^{r}$ for some $r \in R$, and replacing $K$ by $K^{r}$, we may assume $H \leq K$. Then $H$ acts on $X=U \cap K$, so by Lemma 1.1, $X=\prod_{\alpha \in \Phi_{X}}\left(U_{\alpha} \cap X\right)$. If $\alpha \in \Phi_{R}$, then $U_{\alpha} \cap X=U_{\alpha} \cap R \cap X=U_{\alpha} \cap Q \leq Q$, while if $\alpha \in \Phi_{X}-\Phi_{R}$, then $U_{\alpha} \leq L$. Therefore $X \leq L Q$.

Let $w_{0}$ be the long word in the Weyl group of $G$, and $Y=U^{w_{0}} \cap K$. Then $Y$ is $H$-invariant, so by Theorem 1.5, $Y=\prod_{\beta \in \Phi_{Y}}\left(Y \cap U_{\beta}\right)$, so as in the previous paragraph, $Y \leq L$. Therefore $K=\langle H X, Y\rangle \leq L Q$, so $K=L Q$.

Remark. One consequence of Lemma 1.6 is that if $M$ is a maximal subgroup of $P$, then either $R \leq M$ and $M / R$ is maximal in $P / R$ or $M=L R_{0}$, where $R_{0}$ is maximal in $R$ subject to $R \neq R_{0} \unlhd P$. In Theorem 2 in A, we show that if $P$ is a maximal parabolic, then, in the latter case, $R_{0}$ is uniquely determined. In particular, this supplies a description of the subgroups of $G$ of depth 2 contained in a proper parabolic.

Remark. When $q>4$ the fact that $G$ is of Seitz type can be used to show that either the $p$-part of the Schur multiplier of $G$ is trivial or $p=3$ and $G$ is $L_{2}(9)$. This follows from an argument of Ward in [W], using his lemma on Schur multipliers proved in $[\mathrm{W}$. The condition $q>4$ is necessary to insure that the action of $H$ on root groups is not self dual. We will see that when $q>4$ the proof of Theorem 1 is relatively easy; still this gives yet another example of the utility of the Seitz condition.

\section{The Proof of Theorem 1}

We begin the proof of Theorem 1, which involves a series of reduction. Assume $G$ is a counterexample to Theorem 1 and for $\alpha \in \Phi$, set $L_{\alpha}=\left\langle U_{\alpha}, U_{-\alpha}\right\rangle$. In particular, $G$ is not of Seitz type and

Lemma 2.1. $q>2$.

Lemma 2.2. There exist distinct $\alpha, \beta \in \Phi^{+}$such that one of the following holds:

(1) $C_{H}\left(U_{\alpha} / \Phi\left(U_{\alpha}\right)\right)=C_{H}\left(U_{\beta} / \Phi\left(U_{\beta}\right)\right)$.

(2) $C_{H}\left(Z\left(U_{\alpha}\right)\right)=C_{H}\left(Z\left(U_{\beta}\right)\right)$.

(3) $\Phi\left(U_{\alpha}\right) \neq 1 \neq \Phi\left(U_{\beta}\right)$ and $C_{H}\left(U_{\alpha}\right)=C_{H}\left(Z\left(U_{\beta}\right)\right)$.

Proof. If the lemma fails, then (Se2) is satisfied, so as $G$ is not of Seitz type, (Se1) fails, so that there exists $\alpha \in \Phi^{+}$such that $H$ is not irreducible on $U_{\alpha} / \Phi\left(U_{\alpha}\right)$ or $Z\left(U_{\alpha}\right)$. Now $L_{\alpha} / Z\left(L_{\alpha}\right) \cong L_{2}\left(q^{e}\right)$ for some $e \in\{1,2,3\}, U_{3}(q)$, or ${ }^{2} B_{2}(q)$. Further, $q>2$ by Lemma 2.1, so by inspection, $H \cap L_{\alpha}$ is irreducible on the two sections. 
Notation 2.3. In the remainder of the section, choose $\alpha, \beta \in \Phi^{+}$as in Lemma 2.2. Let $\Sigma=\langle\alpha, \beta\rangle$ be the subsystem of $\Phi$ generated by $\alpha$ and $\beta$; that is, $\Sigma=\alpha^{W_{L}} \cup \beta^{W_{L}}$, where $W_{L}=\left\langle s_{\alpha}, s_{\beta}\right\rangle \leq W$ and $s_{\gamma}$ is the reflection determined by $\gamma \in\{\alpha, \beta\}$. For $\Lambda \subseteq \Phi$ write $\mathbf{R} \Lambda$ for the set of roots in the $\mathbf{R}$-linear span of $\Lambda$. Set $L=\left\langle L_{\alpha}, L_{\beta}\right\rangle$. For $\gamma \in\{\alpha, \beta\}$, set $V_{\gamma}=U_{\gamma}$ in Lemma 2.2(1), $V_{\gamma}=Z\left(U_{\gamma}\right)$ in Lemma 2.2(2), and $V_{\alpha}=U_{\alpha}$ and $V_{\beta}=Z\left(U_{\beta}\right)$ in Lemma 2.2(3). Then set $J_{\gamma}=\left\langle V_{\gamma}, V_{-\gamma}\right\rangle$ and $H_{\gamma}=C_{H}\left(V_{\gamma}\right)$. Recall $L_{\gamma}=J_{\gamma}$ unless $\Omega$ is an odd dimensional orthogonal group, $\gamma$ is long, and $V_{\gamma}=Z\left(U_{\gamma}\right)$, where $L_{\gamma} \cong(S) U_{3}(q)$ and $J_{\gamma} \cong S L_{2}(q)$.

Lemma 2.4. Let $\Delta$ be a subsystem of $\Phi$ and let $\gamma, \delta$ be independent roots in $\Phi$. Then:

(1) If $\Delta=\mathbf{R} \Delta$, then any simple system for $\Delta$ is contained in a simple system for $\Phi$.

(2) There exists a simple system $\pi$ for $\Phi$ such that $\gamma \in \pi$ and there is $\epsilon \in \pi$ with $\delta \in \Phi^{+} \cap\langle\gamma, \epsilon\rangle$, where $\Phi^{+}$is the positive system of $\pi$.

(3) If $\Delta=\mathbf{R}\{\gamma, \delta\}$, then there is a simple system $\pi_{\Delta}$ for $\Delta$ and a simple system $\pi$ for $\Phi$ such that $\gamma \in \pi_{\Delta} \subseteq \pi$ and $\delta \in \Phi^{+}$.

Proof. Part (1) is 2.1 in [St, while (2) is Lemma 1 in [Ch]. Then (3) follows from (1) and (2).

Lemma 2.5. Assume $\Sigma$ is of type $A_{1}+A_{1}$. Then:

(1) $q=3$ and either $L_{\alpha} / Z\left(L_{\alpha}\right) \cong L_{\beta} / Z\left(L_{\beta}\right) \cong L_{2}(3)$ or $L / Z(L) \cong U_{4}(3)$, $\alpha$ and $\beta$ are short, and $A_{u t}{ }_{H}(L)$ is not $P_{G} U_{4}(3)$.

(2) $\Omega$ is not an odd dimensional orthogonal group.

Proof. Set $\Delta=\mathbf{R} \Sigma$. Then $\Delta$ is a rank 2 root system and by Lemma 2.4(1) there is a simple system $\pi_{\Delta}$ of $\Delta$ contained in $\pi$. Thus either $\Sigma=\Delta$ or $\Delta$ is of type $B_{2}, C_{2}$ or $G_{2}$, or $W(\Delta) \cong D_{16}$. In the first case, $\left[L_{\alpha}, L_{\beta}\right]=1$, and in the remaining cases we can take $\Delta=\Phi$ and either $G=\Omega$ or $G=\Omega H \cong G U_{4}(3)$. Then, calculating in $G$, we find that either $\left[L_{\alpha}, L_{\beta}\right]=1$ or $L=\Omega \cong S p_{4}(q), S U_{4}(q), S U_{5}(q)$, or ${ }^{2} F_{4}(q)$, with $\alpha$ and $\beta$ short unless $L=S U_{5}(q)$ or $q$ is odd and $L$ is symplectic. For example, some of these calculations appear in the proof of Theorem 2.6.

By Lemma 1.1, $q>2$, so either $K_{\alpha}=H \cap L_{\alpha}$ is nontrivial on $U_{\alpha} / \Phi\left(U_{\alpha}\right)$ and $Z\left(U_{\alpha}\right)$ or $q=3$ and $L_{\alpha} / Z\left(L_{\alpha}\right) \cong L_{2}(3)$. Thus if $\left[L_{\alpha}, L_{\beta}\right]=1$, then $K_{\alpha} \leq C_{H}\left(U_{\beta}\right)$, so (1) holds from the choice of $\alpha, \beta$ in Notation 2.3. Thus we may assume $\left[L_{\alpha}, L_{\beta}\right] \neq$ 1 , so $\Delta=\Phi$ and $L H=G$.

Suppose $J_{\gamma}=L_{\gamma}$ for $\gamma \in\{\alpha, \beta\}$. Then as $L H=G$ and $H_{\alpha}=H_{\beta}$ we have $H_{\alpha}=Z(G)$, which forces (1) to hold; for example, $H_{\alpha}$ is often determined during the proof of Lemma 2.6.

Finally suppose $J_{\gamma} \neq L_{\gamma}$ for some $\gamma \in\{\alpha, \beta\}$. Then $G \cong S U_{5}(q)$, Lemma 2.2(1) does not hold, $\alpha$ and $\beta$ are long, and $\left[L_{\alpha}, J_{\beta}\right]=1$. Therefore $K_{\alpha}$ centralizes $Z\left(U_{\beta}\right)$, so $C_{H}\left(Z\left(U_{\beta}\right)\right)$ does not centralize $Z\left(U_{\alpha}\right)$, a contradiction as Lemma 2.2(1) does not hold.

Lemma 2.6. (1) If $\Sigma$ is of type $A_{2}$, then $q=4, L / Z(L) \cong L_{3}(4)$, and $H$ induces inner automorphisms on $L$.

(2) $\Sigma$ is not of type $B_{2}$ or $C_{2}$.

(3) $\Omega$ is not $G_{2}(q),{ }^{3} D_{4}(q)$, or ${ }^{2} F_{4}(q)$. 
Proof. Set $J=\left\langle J_{\alpha}, J_{\beta}\right\rangle$. For $\gamma \in\{\alpha, \beta\}$ set $K_{\gamma}=H_{\gamma} \cap J$. By choice of $\alpha, \beta$ in Notation 2.3, we have $H_{\gamma}=H_{\alpha}$, so as $H_{\gamma}=C_{H}\left(V_{\gamma}\right)=C_{H}\left(J_{\gamma}\right)$, it follows that $H_{\alpha}$ centralizes $J$. Therefore $C_{H \cap J}\left(J_{\gamma}\right)=K_{\gamma}=Z(J)$.

Suppose first that $\Sigma$ is of type $A_{2}$ so that $L=J \cong S L_{3}(r)$, where $r=q$ or $q^{2}$. As $C_{H \cap L}\left(L_{\alpha}\right)=Z(L)$ from the previous paragraph, and $|Z(L)|=(r-1,3)$, while $K_{\alpha} \cong \mathbf{Z}_{r-1}$ and $q \neq 2$, we conclude that $q=r=4$, so that (1) holds.

Suppose next that $\Sigma$ is of type $B_{2}$ or $C_{2}$. As $\Sigma=\alpha^{W_{L}} \cup \beta^{W_{L}}, \alpha$ and $\beta$ have different lengths, so we may take $\alpha$ long and $\beta$ short. Further, we may take $L$ to be $S p_{4}(q), S U_{4}(q)$, or $S U_{5}(q)$.

Assume $L$ is $S p_{4}(q)$. Then $L=J$ with $|Z(L)|=(q-1,2)$ and $K_{\gamma} \cong \mathbf{Z}_{q-1}$ if $q$ is even or $\gamma$ is short, and $\mathbf{Z}_{2} \times \mathbf{Z}_{q-1}$ otherwise. As $K_{\gamma}=Z(L)$ and $q>2$, we conclude that $q=3$ and $\gamma$ is short, whereas $\alpha$ is long from the previous paragraph, a contradiction.

Therefore $L$ is unitary, so as $\beta$ is short, $L_{\beta} \cong S L_{2}\left(q^{2}\right)$, and hence $\left|H: H_{\beta}\right| \geq$ $\left(q^{2}-1\right) /(q+1,2)$. But if $J_{\alpha} \cong S L_{2}(q)$, then $\left|H: H_{\alpha}\right| \leq q-1$, so as $H_{\alpha}=H_{\beta}, q+1 \leq$ $(q+1,2) \leq 2$, a contradiction. Therefore $\Omega$ is an odd dimensional unitary group and $J_{\alpha}=L_{\alpha} \cong S U_{3}(q)$. Also we may take $G=L=S U(V)$, where $V$ is a 5-dimensional unitary group over $F=\mathbf{F}_{q^{2}}$. We may choose a basis $X=\left\{x_{0}, x_{1}, x_{2}, y_{1}, y_{2}\right\}$ such that $\left\langle x_{1}, x_{2}\right\rangle$ and $\left\langle y_{1}, y_{2}\right\rangle$ are totally singular with $y_{3-i}$ orthogonal to $x_{i}$ and $x_{0}$ nonsingular and orthogonal to the remaining members of $X$, and we may choose $H$ to be the subgroup of $G$ acting on $F x$ for each $x \in X$. Further, we may choose $\left[V, L_{\alpha}\right]=\left\langle x_{0}, x_{2}, y_{2}\right\rangle$. Write $h\left(a_{0}, a_{1}, a_{2}, b_{1}, b_{2}\right)$ for the member of $H$ with eigenvalue $a_{i}$ on $x_{i}$ and $b_{i}$ on $y_{i}$. Then $H_{\alpha}=\left\{h\left(a, b, a, b^{-q}, a\right): b \in F^{\#}, a \in F_{q+1}, a^{3}=b^{q-1}\right\}$, where $F_{q+1}$ is the subgroup of $F^{\#}$ of order $q+1$. Thus $\left|H_{\alpha}\right|=q^{2}-1$. But we saw that $H_{\alpha}=Z(G)$ is of order $(q+1,5)$, so $q^{2}-1 \leq(q+1,5) \leq 5$, contradicting $q \geq 3$. This completes the proof of (2).

Finally, assume $\Omega$ is $G_{2}(q),{ }^{3} D_{4}(q)$, or ${ }^{2} F_{4}(q)$; we may assume $G=\Omega$. Then $\Phi$ is of type $G_{2}$ in the first two cases and $W \cong D_{16}$ in the third. Further, there exists $\gamma_{i} \in \Phi$ such that, setting $L_{i}=L_{\gamma_{i}}$ and $H_{i}=H \cap L_{i}$, we have $\left[L_{1}, L_{2}\right]=1$ and $H_{i}=C_{H}\left(L_{3-i}\right)$. Further, in the first two cases $\gamma_{1}$ is long and $\gamma_{2}$ is short, while in the third case $\gamma_{1}$ and $\gamma_{2}$ are long. Moreover, either $H=H_{1} \times H_{2}$ or $\Phi$ is of type $G_{2}, q$ is odd, and $\left|H: H_{1} H_{2}\right|=2=\left|H_{1} \cap H_{2}\right|$. Finally, if $G$ is ${ }^{2} F_{4}(q)$, then $L_{\alpha} \cong S L_{2}(q)$ or $S z(q)$, so $H_{\alpha} \cong \mathbf{Z}_{q-1}$, while if $\Phi$ is of type $G_{2}$ we may choose $L_{1} \cong S L_{2}(q)$ and $L_{2} \cong S L_{2}(q)$ or $S L_{2}\left(q^{3}\right)$ for $G \cong G_{2}(q)$ or ${ }^{3} D_{4}(q)$, respectively. Therefore as $Z(G)=1$, it follows that $J \neq G$, so $\Sigma \neq \Phi$. If $\Sigma$ is of type $A_{1}+A_{1}$, then by Lemma $2.5, q=3$ and $L_{\alpha} \cong L_{\beta} \cong S L_{2}(3)$, so that $G$ is $G_{2}(3)$, contrary to the choice of $G$ as a counterexample to Theorem 1. It follows that $\Phi$ is of type $G_{2}$ and $\Sigma$ is of type $A_{2}$. Then by (1), $q=4$ and $J=\left\langle U_{\sigma}: \sigma \in \Sigma\right\rangle \cong S L_{3}(4)$. But now $G \cong G_{2}(4)$, contrary to the choice of $G$ as a counterexample to Theorem 1 . This completes the proof of the lemma.

Lemma 2.7. $\Phi$ is of rank at least 3 .

Proof. From Lemma 2.2 the rank is at least 2, so we may assume $\Phi$ is of rank 2 . Thus $\Phi$ is of type $A_{2}, B_{2}, C_{2}$, or $G_{2}$, or $\Omega$ is ${ }^{2} F_{4}(q)$. If $\Phi$ is of type $G_{2}$, then $\Omega$ is $G_{2}(q)$ or ${ }^{3} D_{4}(q)$, so it follows from Lemma 2.6(3) that $\Phi$ is of type $A_{2}, B_{2}$, or $C_{2}$. Therefore $\Phi \neq \Sigma$ by parts (1) and (2) of Lemma 2.6 and the choice of $G$ as a counterexample to Theorem 1. However, if $\Phi$ is of type $A_{2}$, then $\Phi$ has no proper rank 2 subsystems, so we conclude that $\Phi$ is of type $B_{2}$ or $C_{2}$. Then as $\Phi \neq \Sigma, \Sigma$ is 
of type $A_{1}+A_{1}$ and hence $\alpha$ and $\beta$ are of the same length. By Lemma 2.5, $q=3$ and $L / Z(L) \cong L_{2}(3) \times L_{2}(3)$ or $U_{4}(3)$, so $\Omega / Z(\Omega)$ is $P S p_{4}(3)$ or $U_{4}(3)$. As $G$ is a counterexample to Theorem 1 , we conclude that $A u t_{G}(\Omega) \cong P G U_{4}(3)$, so $\alpha$ and $\beta$ are long by Lemma 2.5. But then $H_{\alpha} \neq H_{\beta}$, a contradiction.

Lemma 2.8. $\Sigma$ is not of type $A_{1}+A_{1}$.

Proof. Assume otherwise. We may assume $Z(\Omega)=1$. From Lemma 2.5, $q=3$ and $L / Z(L) \cong L_{2}(3) \times L_{2}(3)$ or $U_{4}(3)$. As $G$ is a counterexample, $\Omega$ is not $P S p_{n}(3)$ with $n \geq 4, P \Omega_{m}^{\epsilon}(3)$ with $m \geq 7$, or $F_{4}(3)$.

Suppose $\Omega$ is $L_{n}^{\epsilon}(3)$. By Lemma 2.7, $n \geq 4$ if $\epsilon=1$ and $n \geq 6$ if $\epsilon=-1$. If $\Omega$ is $L_{4}(3)$, then as $G$ is a counterexample, $G \cong P G L_{4}(3)$. Thus in this case, and when $n \geq 5$, some element of $H$ induces an outer automorphism on $L_{\alpha}$ centralizing $L_{\beta}$, contrary to the choice of $\alpha$ and $\beta$.

Let $\Delta=\mathbf{R} \Sigma$. By Lemma 2.4(3) there is a simple system $\pi_{\Delta}$ for $\Delta$ and a simple system $\pi$ for $\Phi$ with $\alpha \in \pi_{\Delta} \subseteq \pi$. Suppose $\Omega$ is $E_{n}(3)$ for some $n$. As $\pi_{\Delta} \subseteq \pi, \Delta$ is of type $A_{1}+A_{1}$ or $A_{2}$, so as $\Sigma$ is of type $A_{1}+A_{1}$, and $\Sigma \subseteq \Delta$, we conclude that $\Sigma=\Delta$. Then we may take $\pi_{\Delta}=\{\alpha, \beta\}$, so we may take $\alpha, \beta \in \pi$. Then $\alpha, \beta \in \Lambda$ is a subsystem of type $A_{l}, l \geq 4$, generated by members of $\pi$, contrary to the previous paragraph.

This leaves the case where $\Omega$ is ${ }^{2} E_{6}(3)$. As $L / Z(L) \cong L_{2}(3) \times L_{2}(3)$ or $U_{4}(3)$, arguing as in the previous paragraph using Lemma 2.4(3), either $\Sigma=\Delta$, $\alpha$ and $\beta$ are orthogonal long roots and we may take $\alpha, \beta \in \pi$, or $\Delta$ is of type $B_{2}$ or $C_{2}$. The former case yields a contradiction, as the long roots in $\pi$ are not orthogonal. In the latter, $L H$ is contained in a Levi factor $M$ of a parabolic with $M^{\infty} \cong S U_{6}(3)$, and then paragraph two applied to $H M$ supplies a contradiction.

Lemma 2.9. $\Sigma$ is not of type $A_{2}$.

Proof. Assume otherwise. We may assume $Z(\Omega)=1$. By Lemma 2.6, $q=4$, $L / Z(L) \cong L_{3}(4)$, and $H$ induces inner automorphisms on $L$. Further, $\mathbf{R} \Sigma=\Sigma$, so by Lemma 2.4(3), there are simple systems $\pi_{\Sigma}$ and $\pi$ of $\Sigma$ and $\Phi$ with $\alpha \in \pi_{\Sigma} \subseteq \pi$.

Suppose $\pi_{\Sigma}$ is contained in an $A_{3}$-subdiagram $\Delta$ of $\pi$ and set $X=\left\langle U_{\delta}: \delta \in \Delta\right\rangle$. By induction on the order of $G$, we may take $\pi=\Delta$ and $G=X$ so that $G \cong L_{4}(4)$. But then $L H$ is a Levi factor of a parabolic stabilizing a point in the natural module for $G$, so that $A u t_{H L}(L) \cong P G L_{3}(4)$, contrary to paragraph one.

Therefore no such subdiagram exists. But by Lemma 2.7, the rank of $\Phi$ is at least 3 , so $\pi_{\Sigma}$ is contained in a $B_{3}$ or $C_{3}$ subdiagram $\Delta$, and by induction on the order of $G, \pi=\Delta$ and $G=\Omega$. Then $G \cong S p_{6}(4), U_{6}(4), U_{7}(4)$, or $\Omega_{8}^{-}(4)$. As $L / Z(L)$ is $L_{3}(4)$, rather than $L_{3}(16), G$ is not a unitary group. In the remaining cases, $L H$ is the Levi factor in a parabolic stabilizing a totally singular 3 -subspace in the natural module for $G$, so $A u t_{H L}(L) \cong P G L_{3}(4)$, again a contradiction.

We are now in a position to obtain a contradiction, establishing Theorem 1 . Set $\Delta=\mathbf{R} \Sigma$; then $\Delta$ is a rank 2 subsystem of $\Phi$, and by Lemma 2.4(3), we may choose $\pi_{\Delta}$ and $\pi$ as in that lemma. By Lemma $2.7, \Phi$ has rank at least 3 , so $\Delta$ is of type $A_{1}+A_{1}, A_{2}, B_{2}$, or $C_{2}$. By Lemma 2.6(2), Lemma 2.8, and Lemma 2.9, $\Sigma \neq \Delta$. We conclude that $\Delta$ is of type $B_{2}$ or $C_{2}$ and $\Sigma$ is of type $A_{1}+A_{1}$, contrary to Lemma 2.8. This completes the proof of Theorem 1. 


\section{REFERENCES}

[A] M. Aschbacher, Overgroup lattices in finite groups of Lie type containing a parabolic, J. Algebra 382 (2013), 71-99. MR3034474

[Ch] C. Chevalley, Sur certains groupes simples, Tôhoku Math. J. (2) 7 (1955), 14-66 (French). MR0073602 (17,457c)

[CPS] E. Cline, B. Parshall, and L. Scott, Minimal elements of $I(H ; p)$ and conjugacy of Levi complements in finite Chevalley groups, J. Algebra 34 (1975), 521-523. MR.0372061 (51 \#8278)

[Se1] Gary M. Seitz, Small rank permutation representations of finite Chevalley groups, J. Algebra 28 (1974), 508-517. MR0444791 (56 \#3138)

[Se2] Gary M. Seitz, Subgroups of finite groups of Lie type, J. Algebra 61 (1979), no. 1, 16-27, DOI 10.1016/0021-8693(79)90302-8. MR554848(81d:20039)

[Se3] Gary M. Seitz, The root subgroups for maximal tori in finite groups of Lie type, Pacific J. Math. 106 (1983), no. 1, 153-244. MR694680 (84g:20085)

[St] Robert Steinberg, Generators, relations and coverings of algebraic groups. II, J. Algebra 71 (1981), no. 2, 527-543, DOI 10.1016/0021-8693(81)90193-9. MR630615 (83d:14025)

[W] H. N. Ward, On the triviality of primary parts of the Schur multiplier, J. Algebra 10 (1968), 377-382. MR0232858 (38 \#1181)

Department of Mathematics, California Institute of Technology, Pasadena, CaliFORNIA 91125 